\title{
Bone Metastases in Metastatic Renal Cell Carcinoma: Now We Know That Cabozantinib Targets Bone Microenvironment
}

\author{
Raffaele Ratta ${ }^{1}$, Daniele Santini ${ }^{2}$, Elena Verzoni ${ }^{1}$ and Giuseppe Procopio*1 \\ ${ }^{1}$ Department Medical Oncology, Fondazione IRCCS Istituto Nazionale dei Tumori, via G. Venezian 1, 20133, Milan, Italy \\ ${ }^{2}$ Department Medical Oncology, University Campus Bio-Medico of Rome, via Alvaro del Portillo 200, 00128, Rome, Italy
}

${ }^{*}$ Corresponding author: Giuseppe Procopio, Department Medical Oncology, Fondazione IRCCS Istituto Nazionale dei Tumori, via G. Venezian 1, 20133, Milan, Italy, E-mail: giuseppe.procopio@istitutotumori.mi.it

Received date: January 27, 2018; Accepted date: February 08, 2018; Published date: February 16, 2018

Citation: Ratta R, Santini D, Verzoni E, Procopio G (2018) Bone Metastases in Metastatic Renal Cell Carcinoma: Now We Know That Cabozantinib Targets Bone Microenvironment. J Clin Exp Nephrol Vol.3 No.1: 03. DOI: 10.21767/2472-5056.100054

Copyright: @2018 Ratta R, et al. This is an open-access article distributed under the terms of the Creative Commons Attribution License, which permits unrestricted use, distribution, and reproduction in any medium, provided the original author and source are credited.

Keywords: Bone metastases; Bone turnover markers; Cabozantinib; Meteor trial; Renal carcinoma

\section{Commentary}

The multiple tyrosine kinases inhibitor cabozantinib has shown improvements in progression-free survival (PFS), overall survival (OS) and objective response rate (ORR) in comparison to everolimus in patients with metastatic renal cell carcinoma ( $\mathrm{mRCC}$ ) in the METEOR trial, a phase III, randomized, open-label trial $[1,2]$, leading to its approval in $\mathrm{mRCC}$ patients previously treated with antiangiogenic agents.

Escudier and colleagues in their recent paper [3] presented the subgroup analysis of patients with bone involvement treated within the METEOR trial. This analysis underlined the impressive activity of cabozantinib also in patients with bone metastases in all the efficacy endpoints. For patients with bone metastases, median PFS was 7.4 months in the cabozantinib group and 2.7 months in the everolimus group; cabozantinib showed its superiority in patients with bone metastases who also had visceral metastases (median PFS was 5.6 months for the cabozantinib group and 1.9 months for the everolimus group, HR $0.26,95 \% \mathrm{Cl} 0.16-0.43)$. Patients with bone metastases treated with cabozantinib had also an improved ORR in comparison to everolimus: objective responses per independent radiology committee were observed in $17 \%$ of patients treated with cabozantinib with bone metastases and in $20 \%$ of patients with both bone and visceral metastases, while no responses were observed in patients with bone metastases treated with everolimus. Median OS for patients with bone metastases was 20.1 months in the cabozantinib group and 12.1 months in the everolimus group (HR $0.54,95 \% \mathrm{Cl} 0.34-0.84$ ). The superiority of cabozantinib in terms of OS was clear also in patients with bone and visceral metastases (20.1 vs. 10.7 months, $\mathrm{HR} 0.45,95 \% \mathrm{Cl}$ 0.28-0.72). Also bone turnover markers bone-specific alkaline phosphatase (BSAP), N-terminal propeptide of type 1 collagen (P1NP) and C-terminal cross-linked telopeptides of type 1 collagen (CTX) were evaluated during treatment at specific timepoints (at day 1 before the first dose, at week 5 and at week
9) in both patients with and without bone metastases. In patients treated with cabozantinib a greater decrease in P1NP and CTx levels was observed in comparison to patients treated with everolimus; these changes were observed also in patients without bone metastases, suggesting that may be related to a potential effect of cabozantinib on bone microenvironment.

The analysis is of course of primary importance, since bone is not an uncommon site of metastatization: bone metastases (BMs) occur in almost 35\% of patients with advanced RCC and have a negative impact on clinical outcomes [4]. Tyrosine kinase inhibitors (TKIs) have improved survival of patients with mRCC, but they have a limited impact and fewer efficacies on outcomes of patients with BMs in comparison to patients without bone involvement [5].

The strong evidence of the clinical efficacy of cabozantinib on outcomes of patients with BMs is strength and a promising aspect of this drug.

So, which could be the biological mechanisms at the basis of the "osteotropism" of cabozantinib?

First of all, the inhibition of the receptor tyrosine kinase cMET. Patients with tumors expressing high levels of c-MET had significantly shorter PFS and OS than patients with low c-Met levels [6].

The MET and VEGF signaling pathways play a significant role in bone metastatization and regulate the tightly balanced coupling between osteoblasts and osteoclasts, as both these cells express target receptors and are therefore potentially affected by cabozantinib [7]. Osteoblasts and osteoclasts also secrete Hepatocyte Growth Factor (HGF), which is a ligand for cMET, indicating that the HGF/MET signalling axis regulates growth, activity and survival of these cells through autocrine and paracrine mechanisms.

Secondly, cabozantinib could have a direct effect on bone microenvironment [8]: non-cytotoxic doses of cabozantinib significantly inhibit osteoclast differentiation and bone resorption activity and also induce a significant decrease in the expression of receptor activator of nuclear factor kappa-B ligand 
(RANKL) and a concomitant up-regulation of osteoprotegerin levels in mature osteoblasts. Furthermore, c-MET mRNA levels significantly increase in the early stages of osteoclast differentiation, while VEGFR2 is more expressed in mature osteoclasts and both c-MET and VEGFR2 mRNA levels remain unchanged during the osteoblast maturation process. Moreover, when osteoblasts are pre-treated with cabozantinib, they are potentially able to inhibit the osteoclasts differentiation and function.

Finally, the "osteotropism" of cabozantinib has been pointed out by recent data, showing that cabozantinib reduces cell proliferation and migration of four different osteosarcoma cell lines [9].

In conclusion, considering the molecular mechanisms at the basis of bone metastatization, the preclinical and clinical data regarding the bone action of cabozantinib corroborate the theory according to which cabozantinib might be the therapeutic option of choice for mRCC patients and bone involvement.

\section{Conflict of Interest Statement}

Giuseppe Procopio reports receiving fees for serving on advisory boards from Astellas, Bayer, Bristol-Myers Squibb, Ipsen, Janssen, Novartis and Pfizer.

Elena Verzoni reports receiving fees for serving on advisory boards from Ipsen, Janssen, Novartis and Pfizer.

The remaining authors have nothing to disclose.

\section{References}

1. Motzer RJ, Escudier B, McDermott DF, George S, Hammers HJ, et al. (2015) Cabozantinib vs everolimus in advanced renal cell carcinoma. N Engl J Med 1803-1813.
2. Choueiri TK, Escudier B, Powles T, Tannir NM, Mainwaring PN, et al. (2016) Cabozantinib vs everolimus in advanced renal cell carcinoma (METEOR): final results from a randomised, open-label, phase 3 trial. Lancet Oncol 917-927.

3. Escudier B, Powles T, Motzer RJ, Olencki T, Arén Frontera O, et al. (2018) Cabozantinib, New Standard of Care for Patients With Advanced Renal Cell Carcinoma and Bone Metastases? Subgroup Analysis of the METEOR Trial. J Clin Oncol 8: JCO 2017747352.

4. Santini D, Procopio G, Porta C, Ibrahim T, Barni S, et al. (2013) Natural history of malignant bone disease in renal cancer: final results of an Italian bone metastasis survey. PLoS One 8: e83026.

5. Kalra S, Verma J, Atkinson BJ, Matin SF, Wood CG, et al. (2017) Outcomes of Patients With Metastatic Renal Cell Carcinoma and Bone Metastases in the Targeted Therapy Era. Clin Genitourin Cancer 15: 363-370.

6. Peltola KJ, Penttilä $P$, Rautiola J, Joensuu $H$, Hänninen $E$, et al. (2017) Correlation of cMet Expression and Outcome in Patients With Renal Cell Carcinoma Treated With Sunitinib. Clin Genitourin Cancer 15: 487-494.

7. Saylor PJ, Armstrong AJ, Fizazi K, Freedland S, Saad F, et al. (2013) New and emerging therapies for bone metastases in genitourinary cancers. Eur Urol 63: 309-320.

8. Fioramonti M, Santini D, luliani M, Ribelli G, Manca P, et al. (2017) Cabozantinib targets bone microenvironment modulating human osteoclast and osteoblast functions. Oncotarget 8: 20113-20121.

9. Fioramonti M, Fausti V, Pantano F (2018) Cabozantinib Affects Osteosarcoma Growth Through A Direct Effect On Tumor Cells And Modifications In Bone Microenvironment. Sci Rep 8. 